\title{
Feste Kombinationen als Mittel der ersten Wahl bei P. Kardos allen Schweregraden des persistierenden Asthmas?
}

\author{
Fixed Combinations as a First Line Treatment for All Degrees of Severity \\ in Asthma?
}

\section{Zusammenfassung}

Die derzeitige Asthma-Leitlinie der Deutschen Atemwegsliga, die 1998 publiziert wurde, sieht für alle Schweregrade - mit Ausnahme des leichten intermittierenden Asthmas - ein inhalatives Kortison (ICS) vor. Die Anwendung von lang wirkenden $\beta_{2}$-Adrenergika (LABA) ist erst ab Stufe drei (mittelschweres persistierendes Asthma) vorgesehen. Ein Kombinationspräparat (LABA und ICS) war damals noch nicht auf dem Markt. Aufgrund der Entwicklung des Absatzes der Kombinationspräparate in den Industrieländern und in Deutschland ist es anzunehmen, dass sie für die Dauertherapie längst als Mittel der ersten Wahl eingesetzt werden. Da die Kombinationspräparate noch neu sind, liegen derzeit keine randomisierten doppelblinden Studien vor, um den Platz dieser hervorragenden Medikamente im Stufenschema des Asthmas evidenzbasiert zu bestimmen. Darüber hinaus wird anhand einer Kasuistik aufgezeichnet, dass die Einstufung nach Schweregraden, und damit die Entscheidung über die Therapie, in der Praxis nicht immer eindeutig und einfach ist. So lässt auch die Akuttherapie Fragen im Hinblick auf den primären Einsatz des Kombinationspräparates offen. Schließlich ist darauf hinzuweisen, dass über die wissenschaftliche Betrachtung hinaus auch zunehmend wirtschaftliche Überlegungen das Handeln der Ärzte im heutigen rationalisierten Gesundheitswesen mitbestimmen müssen.

\section{Abstract}

Beyond the mild intermittent asthma the present German Asthma Guideline, published in 1998 supports the use of ICS for all steps of asthma severity. However, LABA's have only been recommended as an add on treatment for step 3 (moderate persistent) and 4 (severe asthma). At that time, fixed combinations containing LABA and ICS were not on the market yet. The development of sales of the combination product in industrialized countries including Germany shows that they are probably prescribed for first line treatment and for all steps of asthma severity. The role of these recently developed excellent drugs could not yet be defined by principles of evidence based medicine, i.e. by randomized, double-blind controlled trials. A vignette case should demonstrate that in the daily care even grading severity of asthma regarding the prescription of the appropriate treatment can be difficult. Since the costs of the combination treatment are high, nowadays financial considerations increasingly determine medical decisions in the German Health Care System. The consequent debate of an expert protagonist and an expert opponent should take all these aspects into consideration and help primary care and specialist care physicians to prescribe this type of drugs for the "right" patient. 
Die derzeitige Asthmaleitlinie der Deutschen Atemwegsliga, die 1998 publiziert wurde, sieht für alle Schweregrade - mit Ausnahme des leichten intermittierenden Asthmas - ein inhalatives Kortison (ICS) vor. Die Anwendung von lang wirkenden $\beta_{2}$-Adrenergika (LABA) ist erst ab Stufe drei (mittelschweres persistierendes Asthma) vorgesehen [1]. 1999 erschien das erste Kombinationspräparat in Deutschland auf dem Markt. Zunächst lag es an der Hand, dieses Präparat bei Patienten einzusetzen, die nach der aktuellen Leitlinie sowohl ICS als auch LABA benötigen. Hierdurch wurde im Wesentlichen nichts anderes als eine Vereinfachung der Anwendung aus einem einzigen Gerät angestrebt [2]. Die steil ansteigende Verordnung beider sich mittlerweile auf dem Markt befindlichen Kombinationspräparate führte - wenn überhaupt nur zu einem geringen Rückgang der ICS-Verordnungen. Es liegt daher der Verdacht nahe, dass zusätzliche Patienten mit der Kombination behandelt werden, die den Schweregraden 1 und 2 (leichtes intermittierendes und leichtes persistierendes Asthma) angehören. Bei Neufassung der internationalen [3] und manchen nationalen [4] Leitlinien in den letzten zwölf Monaten wurde ein früherer Einsatz der Kombination schon bei Stufe 2 empfohlen.

Unter solchen Umständen stellt sich die dringende Frage, welchen Stellenwert die derzeit unter Revision stehende deutsche Asthmaleitlinie der Kombination einräumen sollte.

Die Verordnung von ICS + LABA auf Stufe 3 der Atemwegsliga oder GINA ist unumstritten, da die Stufen drei und vier sich gerade dadurch auszeichnen, dass eine ICS in Verbindung mit einer gelegentlichen Inhalation von einem kurz wirksamen Bronchodilatator für eine optimale Asthmakontrolle nicht ausreicht. Solche Patienten benötigen die Verordnung eines weiteren Medikamentes, vorzugsweise eines LABA (bzw. zusätzlich systemisches Kortison in Stufe 4). Selbstverständlich können ICS und LABA auch als Kombinationspräparat gegeben werden, die Wirksamkeit ist mindestens genauso gut wie einzeln verordnet [5], die Compliance ist besser. Die Förderung einer guten Therapieadhärenz durch ein Kombinationspräparat, dessen Wirkung im Gegensatz zu dem reinen Langzeiteffekt der ICS durch den Patienten unmittelbar nach der Inhalation erfahrbar ist, darf nicht unterschätzt werden. Zur Diskussion steht daher die Frage, ob Kombinationspräparate bereits auf Stufe 2 oder 1 verordnet werden sollen. Allerdings sollte nach Feststellungen der GINA 2000 eine optimale Kontrolle des Asthmas auf diesen Stufen auch ohne LABA, nur mit ICS erreicht werden können. LeukotrienrezeptorAntagonisten sind eine weitere, wenn auch umstrittene Option zur Behandlung dieser Patientengruppe. Bei der eventuellen Ausweitung der Indikation für die Kombinationspräparate sind zunächst auch höhere Therapiekosten zu berücksichtigen.

Es ist nicht das Thema dieses Beitrags, Argumente für und wider die Anwendung der Kombination zu diskutieren. Tatsache ist, dass der Markt die Frage mittlerweile durch den überproportionalen Anstieg des Anteils der Kombinationspräparate zugunsten derselben bereits entschieden hat. Anhand der folgenden, alltäglichen Kasuistik sollte gezeigt werden, welches Dilemma in der täglichen Praxis bei der Verordnung der „richtigen“ Asthmatherapie entstehen kann.

\section{Kasuistik}

Der 44-jährige männliche Patient (Nichtraucher) erkrankte im Dezember 1999 an einem Erkältungsinfekt mit Husten, Schnupfen und Kopfschmerzen. Die Nacht vor der Vorstellung traten Atemnot und pfeifendes Atemgeräusch auf. Er leidet seit fünf Jahren, immer in den Monaten April, Mai und Juni, an einer intermittierenden allergischen Rhinitis (Heuschnupfen). Die körperliche Untersuchung war unauffällig, insbesondere fanden sich keine Hinweise auf eine Bronchialobstruktion. Tab. 1 zeigt die Daten der Lungenfunktion, vor und nach Gabe von $200 \mu \mathrm{g}$ Salbutamol.

Tab. 1 Lungenfunktion bei der Vorstellung des Patienten

\begin{tabular}{|lcc|}
\hline Parameter & vor Bronchodilatation & nach $200 \boldsymbol{\mu g}$ Salbutamol \\
\hline VC (\%) & 116 & 128 \\
\hline$F E V_{1}(\%)$ & 98 & 115 \\
\hline$F E V_{1} /$ VC (\%) & 66 & 70 \\
\hline
\end{tabular}

Bis auf das Verhältnis $\mathrm{FEV}_{1} / \mathrm{VC}$ (Tiffeneau Index), das leicht erniedrigt ist, sind die Ausgangswerte der Lungenfunktion normal. Zur Heuschnupfenallergie des Patienten könnte die Neigung zur bronchialen Hyperreagibilität passen, die außerhalb der Pollensaison offensichtlich im Rahmen eines viralen Infektes erstmalig als nächtliches Asthma in Erscheinung getreten ist. Nach der normalen Ausgangslungenfunktion handelt es sich allenfalls um ein leichtes, intermittierendes Asthma, Schweregrad 1. Die Einstufung des Schweregrades ist auch über die Symptome wie nächtliches Erwachen schwierig selbst dann, wenn man das Prinzip berücksichtigt, dass jeweils nach dem schlechtesten Parameter (Lungenfunktion, nächtliches Erwachen, Gebrauch von Notfallmedikation) eingestuft werden sollte. Der Patient hat erst einmal nächtliche Beschwerden gehabt. Nach dem nächtlichen Erwachen (nur ein einziges Mal!) besteht Schweregrad 1 - 2. Es stehen folgende Therapieoptionen zur Verfügung:

1. Alleinige Verordnung eines schnell wirkenden Bronchodilatators für den Fall, dass auch während der Folgenächte Asthmaanfälle auftreten. Diese Option ist leitliniengerecht, wenn man den Schweregrad 1 der Verordnung zugrunde liegt. Es ist damit zu rechnen, dass die im Rahmen des Infektes entstandene bronchiale Hyperreagibilität in maximal sechs Wochen spontan abklingen würde. Es gibt in der Literatur einen einzigen Hinweis, gezeigt an einer kleinen Anzahl von Patienten, dass eine ICS-Therapie einer postinfektiösen bronchialen Hyperreagibilität der späteren Entstehung eines Asthma bronchiale möglicherweise entgegenwirken würde [6]. Medikamentenkosten: unter 10 Euro.

2. Verordnung eines ICS zusätzlich zum schnellwirkenden Bronchodilatator für die Dauer von zwei bis drei Wochen. Diese Option wird von den Leitlinien gestützt, wenn man Schweregrad 2 annimmt. Es ist damit zu rechnen, dass nach zwei bis drei Tagen Therapie die nächtlichen Symptome bereits nachlassen würden. Medikamentenkosten: 40 Euro.

3. Verordnung eines Kombinationspräparates (ICS + LABA) zusätzlich zum schnellwirkenden Bronchodilatator. Der Patient 
könnte bereits die der Konsultation folgenden Nacht durchschlafen. Medikamentenkosten: 90 Euro.

\section{Folgerungen für die Praxis}

Die veraltete deutsche Asthma-Leitlinie bietet keine zuverlässige Antwort auf die Frage, ob Kombinationen von ICS und LABA als Mittel der ersten Wahl bei allen Schweregraden des Asthmas eingesetzt werden sollten. In manchen Fällen, wie die Kasuistik zeigt, kann schon die Einstufung des Schweregrades schwierig sein. Letztlich ist der behandelnde Arzt gefragt, aufgrund der Argumente von Protagonisten und Gegnern über die beste individuelle Therapie eines Patienten zu entscheiden. Obwohl das Kombinationspräparat den Vorteil der sofortigen Beschwerdefreiheit verspricht, können finanzielle Überlegungen in unserem rationierten Gesundheitswesen leider nicht mehr außer Acht gelassen werden.

\section{Literatur}

${ }^{1}$ Wettengel R, Berdel D, Hofmann D et al. Empfehlungen für die Therapie des Asthma bei Kindern und Erwachsenen. Pneumologie 1998; 52 (11): $591-601$

2 Buhl R, Kardos P, Magnussen $\mathrm{H}$ et al. Feste Kombination von inhalierbaren Kortikosteroiden und langwirksamen Beta 2 Sympathomimetika für die Langzeittherapie des Asthma bronchiale. Pneumologie 1999; 53 (4): 210-212

${ }^{3}$ Global Initiative on Asthma. http://www.ginasthma.com/.6-17-0003. Ref Type: Elektronisches Zitat.

${ }^{4}$ British Guideline on the Management of Asthma. Thorax 3 A.D.; 58 (Suppl 1): i1 -i83

${ }^{5}$ Zetterström O, Buhl R, Mellem H et al. Efficacy and safety of a new single inhaler product, containing both budesonide and formoterol, in adult asthma. Eur Respir J 2000; 16 (Supplement 31): 455S

${ }^{6}$ Fujimura M, Ogawa H, Nishizawa Y et al. Comparison of atopic cough with cough variant asthma: is atopic cough a precursor of asthma? Thorax 2003; 58 (1): $14-18$

\section{Buchbesprechung}

A. Wüsthof, V. Böhning

„Kippen“. Leben ohne Zigaretten

Urban \& Fischer Verlag München 2002. 76 S. br., 14,95

ISBN 3-437-47460-X

Ein ungewöhnliches Buch - schon äußerlich auffällig durch sein Format (Hochformat, 25,5 × $21 \mathrm{~cm}$ ); es erinnert ein wenig an ein Kinderbuch, auch wegen des festen, fast kartonartigen Papiers. Innen sind die 76 Seiten farbig gestaltet; als erstes fesseln die zum Teil doppelseitigen Bilder von Tabak, von Zigaretten in verschiedenen Stadien während des Rauchens, auch als Kippen, und Photos von meist jugendlichen Menschen.

Eingesprengt sind Texte über die Vorteile, das Angenehme des Rauchens, seine Nachteile und Gefahren. Der Weg, die Verführung zur Zigarette, die Entwöhnung mit ihren Schwierigkeiten, schließlich das Leben ohne Rauchen werden beschrieben, gegliedert in 3 Kapitel: „Rauchen“ - „Aufhören“ - „Nicht rauchen“. Die dargestellten Personen geben in kurzen persönlichen Kommentaren Auskunft darüber, wie sie zum Rauchen kamen, warum sie rauchen, weshalb und wie sie von der Sucht loskamen. Zum Schluss werden in der Rubrik „Kontakte“ wichtige Adressen genannt und weitere wertvolle Hilfen angeboten, schließlich Informationen über die Autoren gegeben.

Diese, die Kommunikations-Designerin Verena Böhning und der Kinderarzt und Medizinjournalist Dr. Achim Wüsthof, verstehen auf unprätentiöse, nüchterne und überzeugende Weise, den Untertitel des Buches „Leben ohne Zigaretten“ verständlich und erstrebenswert darzustellen. Sie wollen Raucher, vorwiegend Jugendliche, zum Nachdenken über ihr Rauchen bringen, Motivationen und Wege, Hilfen zum Aufhören anbieten, das Leben ohne Zigaretten schildern. Im Gegensatz zu den oft als langweilig, predigend, uncool empfundenen Äußerungen von Eltern, Lehrern oder Ärzten ist dieses Buch ohne störenden Fachjargon, interessant, lebendig geschrieben und informativ.

Ein Buch, empfehlenswert zur Raucherentwöhnung, nicht nur für jugendliche Raucher. 